\title{
NUCLEAR DNA AND THE EVOLUTION OF ALLOTETRAPLOID BRASSICAE
}

\author{
S. C. VERMA and H. REES \\ Deportment of Agricultural Botony, U.C.W., Aberystwyth
}

Received 12.ix.73

\section{SumMary}

DNA amounts, by microdensitometry, are underestimated in nuclei with high DNA density. A method is described for correcting the DNA readings in DNA dense nuclei.

DNA measurements in the allotetraploid Brassica napus $(2 n=38)$, B. carinata $(2 n=34)$ and $B$. juncea $(2 n=36)$, after applying the correction, were found to equal the sum of the diploid parents from which they are derived. This finding, contrary to previous reports, refutes the evidence for a nuclear DNA diminution subsequent to the formation of the allotetraploids.

Interphase nuclei in newly synthesised allotetraploids are substantially larger than in long established allotetraploids. It is suggested that the reduction in nuclear size in tetraploids is associated with greater condensation of chromosome material which, in turn, may reflect an adaptive "switching off" of redundant gene copies in the tetraploid complement.

\section{Introduction}

WITH rare exceptions (see Mischke, 1968) the DNA amount per set of chromosomes (the $C$ value) is constant within a species. Thus the haploid set at $G 1$ during interphase is $1 C$, the diploid $2 C$, the autotriploid $3 C$, the autotetraploid $4 C$ and so on. In allopolyploids the nuclei are made up of the chromosome complements of different diploid species and we would expect their nuclear DNA content to equal the sum of the diploid DNA amounts. For example in an allotetraploid, $A A B B$, made up of the diploid sets, $A A$ with $x$ DNA and $B B$ with $y$, the expectation would be a nuclear DNA content of $x+y$. This is the situation reflected by results in the Triticinae (Rees and Walters, 1965; Nishikawa and Furuta, 1969; cf. Pai and Swaminathan, 1960) and in Avenae (Bullen and Rees, 1972). There are, however, reports of DNA values in allopolyploids amounting to less than the sum of the diploid quantities. One of these concerns a Brassica tetraploid, $B$. napus, which derives from the hybridisation of the diploids $B$. campestris and B. oleracea. According to Yamaguchi and Tsunoda (1969) the nuclear DNA amount in the long-established cultivated form of $B$. napus is about 16 per cent less than is predicted by adding the DNA amounts of the diploid progenitors. They concluded that a substantial loss of DNA had taken place subsequent to the original hybridisation. If this is indeed the case the phenomenon is remarkable. We have re-investigated the DNA variation in $B$. napus and its diploid ancestors and examined, as well, the DNA variation in related diploids and allotetraploids within the genus Brassica.

\section{Materials AND methods}

The material investigated is described in table 1 . Combinations of the three diploids, Brassica campestris $(2 n=20), B$. oleracea $(2 n=18)$ and $B$. 
nigra $(2 n=16)$ gave rise to the three allotetraploids, $B$. napus $(2 n=38)$, $B$. carinata $(2 n=34)$ and $B$. juncea $(2 n=36)$ as shown in fig. 1 .

\section{TABLE 1}

The material

\section{Species}

Brassica compestris

Brassica campestris (B. chinesis)

Brassica carinata A. Br.

Brassica juncea (L.) Coss.

Brassica obracea $\mathbf{L}$.

Brassica napus $\mathbf{L}$.

Brassica nigra (L.) Koch
Cultivars

Mammoth Purple Top

Turnip Wallace

Broccoli, Early Purple

Sprouting

Kohl Rabi Green Top

Hungry Gap Kale

Swede, Wilhelmsbarge
Leeds accession number

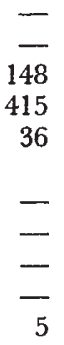

The seeds were kindly supplied by Dr D. J. Harberd of the Dept. of Agricultural Botany, Leeds University, to whom we are grateful.

DNA measurements in root meristem nuclei at $G$ l were made by Feulgen photometry, using an M85 Vickers microdensitometer, following the fixing and staining procedure of McLeish and Sunderland (1961) with one slight modification, namely that the $p \mathrm{H}$ of the Feulgen solution was adjusted to

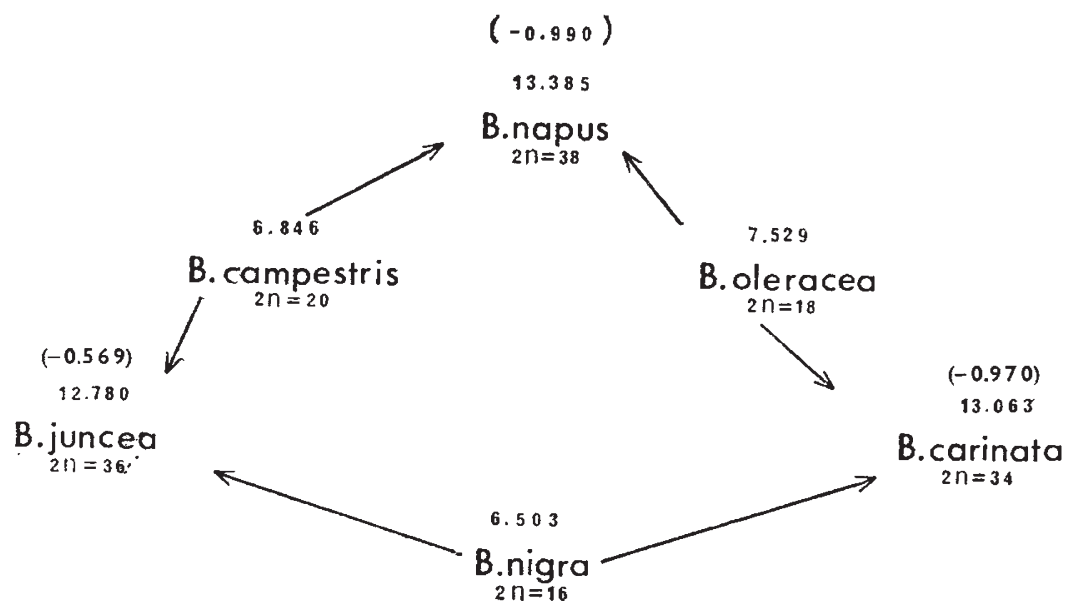

Frg.1.-Mean, uncorrected, DNA values (in arbitrary units) in root meristem nuclei at

$G 1$ in three diploid Brassicas and their allopolyploid derivatives. Values in brackets are deviations from expectation.

$3 \cdot 6$ (see Walker and Richards, 1959). The "DNA density" of each nucleus was calculated by dividing the DNA value by the display area of the nucleus scanned.

\section{Results}

The $G 1$ values for each species are presented in table 2. It is important to point out, first, that there was no significant variation in nuclear DNA amounts between cultivars within species listed in table 1. The table shows 
TABLE 2

Nuclear DNA amounts at G1 in diploid and allotetraploid Brassicae

\begin{tabular}{|c|c|c|c|c|}
\hline \multirow[b]{2}{*}{ Species } & \multicolumn{2}{|c|}{ DNA } & \multirow[b]{2}{*}{ Mean } & \multirow[b]{2}{*}{$\mathrm{DNA} \times 10^{-12}$} \\
\hline & Replicate I & Replicate II & & \\
\hline B. campestris & $\begin{array}{l}6 \cdot 78 \\
6 \cdot 87\end{array}$ & $\begin{array}{l}6 \cdot 73 \\
7 \cdot 01\end{array}$ & $6 \cdot 84$ & $1 \cdot 64$ \\
\hline B. oleracea & $\begin{array}{l}7 \cdot 30 \\
8 \cdot 22\end{array}$ & $\begin{array}{l}7 \cdot 54 \\
7 \cdot 06\end{array}$ & $7 \cdot 53$ & $1 \cdot 81$ \\
\hline B. nigra & $\begin{array}{l}6 \cdot 39 \\
6 \cdot 55\end{array}$ & $\begin{array}{l}6 \cdot 66 \\
6 \cdot 41\end{array}$ & $6 \cdot 50$ & $1 \cdot 56$ \\
\hline B. carinata & $\begin{array}{l}12 \cdot 95 \\
13 \cdot 32\end{array}$ & $\begin{array}{l}13 \cdot 10 \\
12 \cdot 88\end{array}$ & $13 \cdot 06$ & $3 \cdot 13$ \\
\hline B. juncea & $\begin{array}{l}12 \cdot 66 \\
12 \cdot 73\end{array}$ & $\begin{array}{l}12 \cdot 96 \\
12 \cdot 78\end{array}$ & $12 \cdot 78$ & 3.07 \\
\hline B. napus & $\begin{array}{l}13 \cdot 83 \\
13 \cdot 40\end{array}$ & $\begin{array}{l}12 \cdot 96 \\
13 \cdot 35\end{array}$ & $13 \cdot 38$ & $3 \cdot 21$ \\
\hline
\end{tabular}

In each replicate the two DNA values are the means derived from measurements of the nuclei at $G 1$ in one root tip from each of two seedlings. Raw data are in arbitrary units. Absolute amounts are given in the fifth column, following conversion using Allium cepa as a standard $\left(2 C=33.5 \times 10^{-12} \mathrm{~g}\right)$.

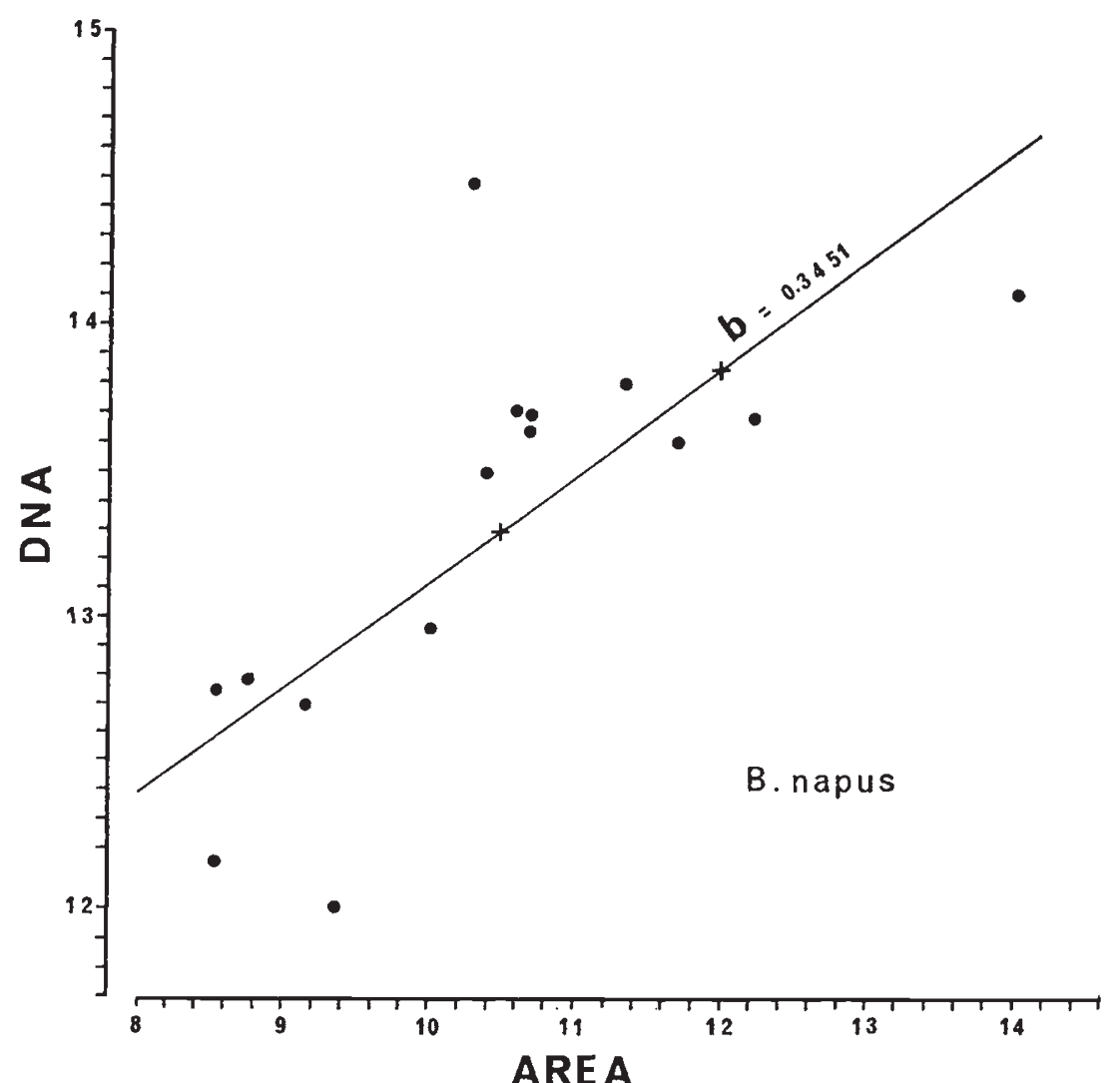

Fig. 2.-DNA readings at $G 1$ plotted against the nuclear areas (both in arbitrary units). 
that the DNA amounts differ between diploid species, between tetraploid species and, of course, between diploids and tetraploids. It will be observed, also, that while the values for tetraploid species approximate to the expected sums of the parental values there is in each case a deviation from expectation. The deviations are consistent in that DNA readings in all the tetraploid species are lower than expected, 7 per cent lower in B. napus. The deviations are highly significant $(P=<0 \cdot 01)$. On the face of it these results confirm and indeed extend the findings of Yamaguchi and Tsunoda (1969), testifying to a DNA diminition in tetraploid species. The following observations on the relationship between the $G$ I DNA readings and the area of the nucleus scanned indicate, however, that the results in table 2 are not strictly valid.

\section{(i) $D \mathcal{N} A$ amount and nuclear $D \mathcal{N} A$ " density"}

In fig. 2 we have plotted the DNA readings against the nucleus area in $G 1$ telophases and interphases of $B$. napus. It will be observed that the DNA readings increase with the area of the nucleus. The regression is significant $(P=<0 \cdot 01)$. The conclusion is that the estimate of DNA amount is dependent upon the DNA density (per unit area) of the nucleus scanned and is underestimated in the smaller nuclei (see Mittwoch, 1969). The conclusion could be questioned on the grounds that all the nuclei are not at $G \mathrm{l}$, that some, the larger nuclei, are in $S$ and therefore have begun DNA synthesis, in which case the increase in DNA reading is real. We doubt whether this objection is valid, because the $G$ l nuclei were selected

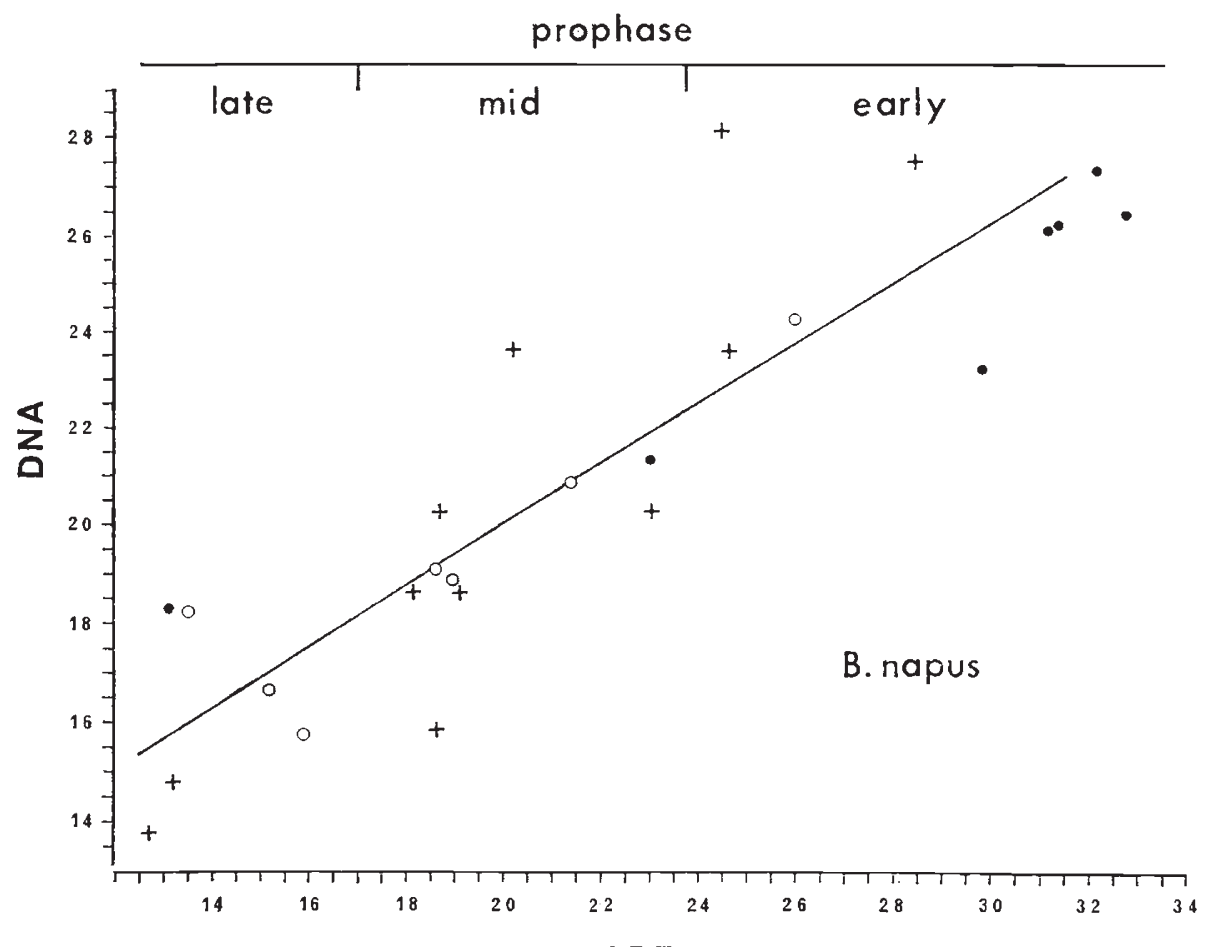

AREA

FIG. 3.-DNA measurements in prophases plotted against areas (arbitrary units). 
with care. Even so we have verified the conclusion as follows. B. napus nuclei at prophase, unquestionably with identical DNA contents, were scanned and the DNA readings plotted against area (fig. 3). It will be observed that the DNA readings increase with increasing area i.e. with decreasing DNA density. There is no doubt, therefore, that the DNA amount is underestimated in the denser nuclei. The conclusion is of particular relevance because the DNA density of tetraploid nuclei is significantly higher than that of diploid nuclei in these Brassicas (fig. 5). The possibility is, therefore, that the DNA deficiency within tetraploids is

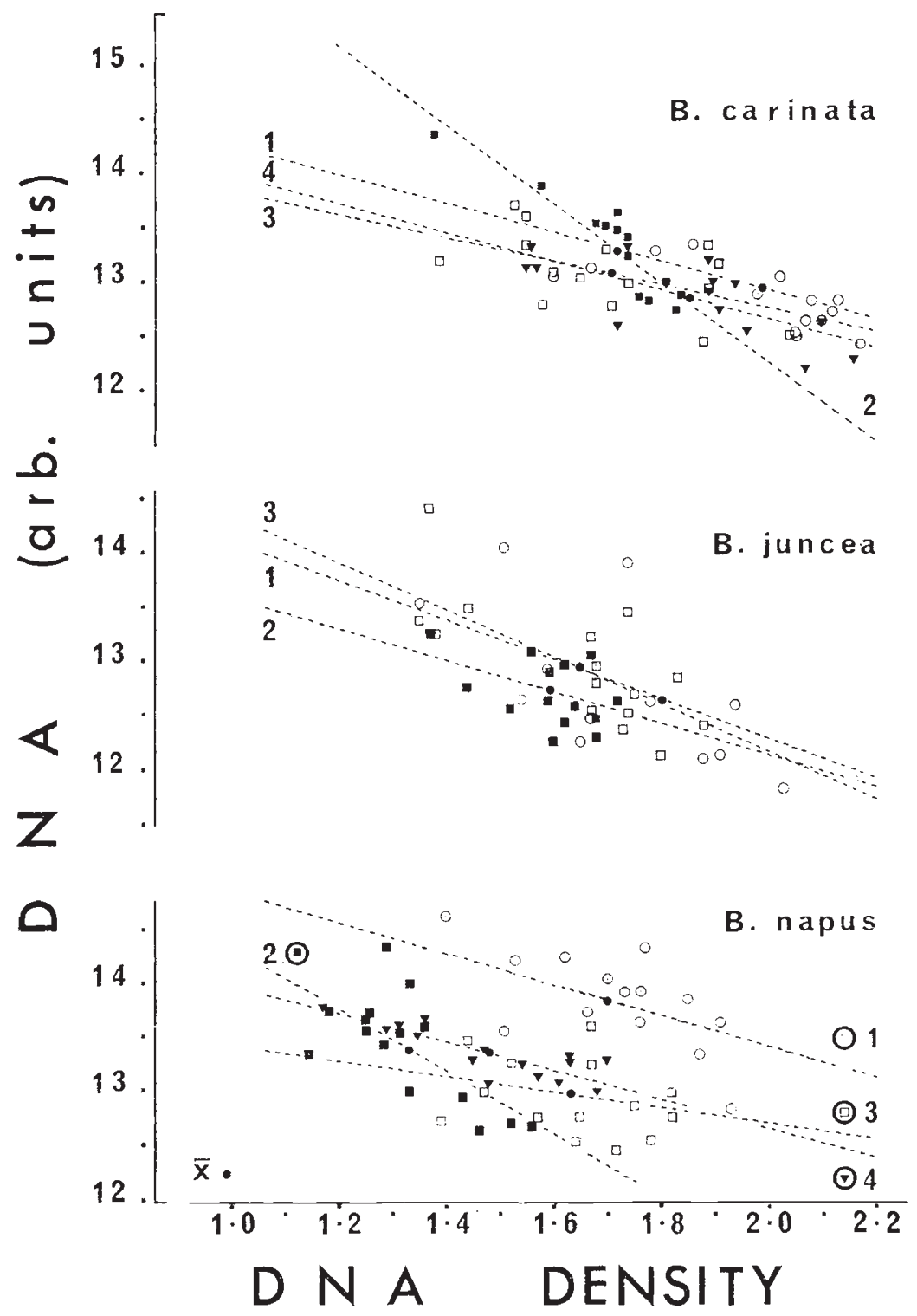

Frg. 4.-DNA reading plotted against area in 15 nuclei in 11 tetraploid plants ( 4 each in $B$. carinata and B. napus, 3 in $B$. juncea).

$33 / 1-\mathbf{E}$ 
accounted for by error, an underestimation of the DNA due to higher nuclear density.

\section{(ii) A correction}

In fig. 4 are DNA readings plotted against density in 15 nuclei per replicate in each tetraploid species. It will be seen that the DNA estimates increase consistently with decreasing density. The joint regression is linear and significant at the 1 per cent level. There is no significant heterogeneity between species. By calculating the slopes of the regressions it is possible to find out the extent to which the tetraploid DNA amounts are " distorted" by virtue of their greater densities. This error may then be used to determine what the tetraploid DNA values would be at densities corresponding with those at which the diploid readings were made.

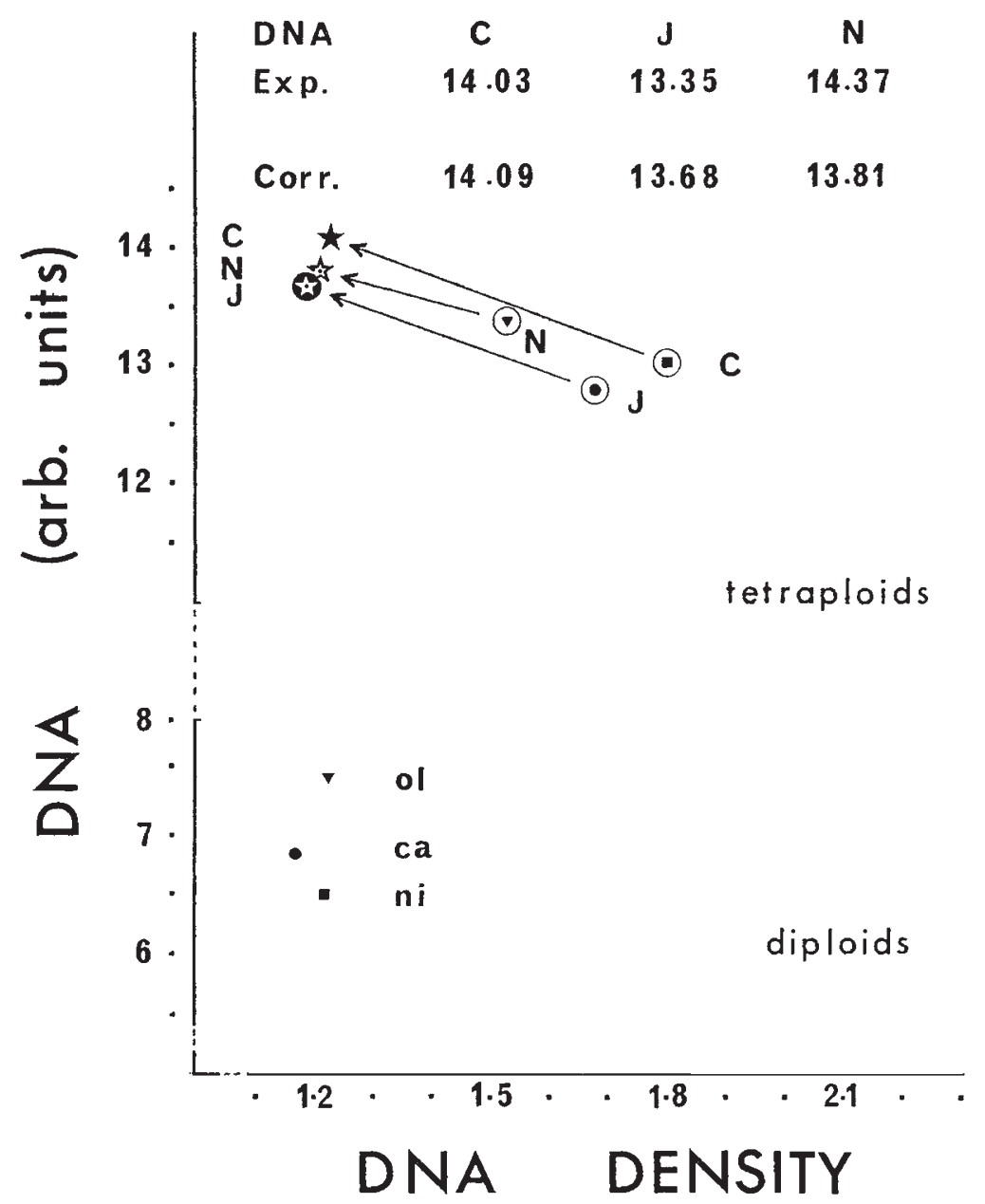

FIg. 5.-DNA values at $G 1$ plotted against the nuclear DNA density. The DNA density for each species was calculated from the average nuclear DNA reading plotted against the average nuclear area. $o l=B$. oleracea, $c a=B$. campestris, $n i=B$. nigra, $C=B$. carinata, $\mathcal{N}=\mathcal{N}$. napus, $\mathcal{F}=B$. juncea. The " corrected " DNA values for the tetraploids are plotted as stars. 
Fig. 5 shows that the corrected tetraploid values fit closely with expectation and analysis confirms they do not deviate significantly from the expected amounts. We conclude that no DNA diminution is associated with allopolyploidy in these Brassicae. We conclude also that previous claims for a DNA diminution were probably based on underestimating the DNA contents of the (relatively) dense tetraploid nuclei.

\section{(iii) Old and new tetraploids}

The results of Yamaguchi and Tsunoda (1969) showed, as we have already mentioned, that the DNA amounts in the cultivated, long-established, allotetraploid $B$. napus were substantially less than the expected sum of the diploid DNA amounts. In marked, and surprising, contrast the DNA amounts given for newly synthesised allotetraploid $B$. napus were much higher and were, in fact, equal to the expected sums of the diploid parents. Our own data for a newly synthesised $B$. napus (table 3 ) were very similar to

\section{TABLE 3}

G1 DNA values (in arbitrary units) by microdensitometry in a long established variety of Brassica napus (Swede Wilhelmsbarge), a newly snythesised $\mathrm{B}$. napus and in the diploid progenitors, B. campestris and $\mathrm{B}$. oleracea. It will be observed that the nuclear DNA values in the resynthesised B. napus are substantially (and significantly) higher
B. campestris (B. chinensis)
$6.75 \pm 0.06$
B. oleracea (Kohl Robi Purple Top)
$7 \cdot 45 \pm 0.07$
$B$. napus (Swede Wilhelmsbarge)
B. napus (resynthesised)*

* This "new" tetraploid was grown from the second seed generation following hybridisation and " doubling " of the chromosome number. These seeds were also supplied by Dr D. J. Harberd of Leeds.

those of Yamaguchi and Tsunoda's. Uncorrected tetraploid values did not depart significantly from the sum of the diploid parent values. Why should the results for new and old tetraploids differ?

Yamaguchi and Tsunoda maintain that nuclear DNA has been lost subsequent to the formation of the older tetraploids. Our evidence shows that no such DNA diminution has taken place. If this is indeed the case the explanation for the difference between old and new tetraploids must lie elsewhere. The nuclear volume in root-tip cells of long-established tetraploids is much smaller (about 60 per cent) than in root tips of newly synthesised tetraploids (Yamaguchi and Tsunoda loc. cit., and our own data) and the DNA density of nuclei therefore much higher. As explained above increasing nuclear DNA density leads to underestimation of the nuclear DNA amount by microdensitometry. The lower DNA readings in old as compared with newly synthesised tetraploids is clearly therefore a direct consequence of the reduced nuclear volume in the former.

The reduction in nuclear size in the old-established tetraploids is, in itself, remarkable. What it signifies in terms of nuclear organisation and function is not clear. It is tempting to speculate that the higher DNA density of nuclei in long-established tetraploids is a reflection of increased 
chromosome condensation at interphase which, in turn, may reflect the switching off of redundant gene copies in the tetraploid complement.

\section{Gonclusion}

We consider that the present evidence disproves the claim that a substantial DNA diminution has taken place since the formation of allotetraploid Brassicae species. On the contrary, the DNA contents of tetraploid nuclei represent the sums of the DNA contents of their diploid ancestors. In this respect the results are not especially remarkable. If nothing else, however, they serve to dispose effectively of a surprising, even embarrassing hypothesis.

\section{REFERENGES}

BUllen, M. R., AND REES, H. 1972. Nuclear variation in Avenae. Chromosoma, 39, 93-100. MCLEISH, J. L., AND SUNDERLAND, N. 1961. Measurements of deoxyribonucleic acid (DNA) in higher plants by Feulgen photometry and chemical methods. Exp. Cell Res., 24, $527-540$.

MIKSCHE, J. P. 1968. Quantitative study of intra specific variation of DNA per cell in Picea glauca and Pinus banksiana. Can. F. Gen. Cyt., 10, 590-600.

MrTTwoch, U. 1969. Scope and limitations of Feulgen microdensitometry. Chromosomes Today, 2, 90-98. Oliver and Boyd, Edinburgh.

NISHIKAWA, K., AND FURATA, Y. 1969. DNA content per nucleus in relation to phylogeny of wheat and its relatives. Fap. F. Gen., 44, 23-29.

PAI, R. A., AND SWAminathan, M. S. 1960. Differential radiosensitivity among the probable genome donors of bread wheat. Evolution, 14, 427-432.

REES, H., AND WALTERS, M. R. 1965. Nuclear DNA and the evolution of wheat. Heredity, 20, 73-82.

WALKER, P. M. B., AND RICHARDS, B. M. 1959. Quantitative microscopical techniques for single cells. The Cell, Vol. 1. Academic Press.

YAMAGUCHI, Y., AND TSUNODA, s. 1969. Nuclear volume, nuclear DNA content and radiosensitivity in Brassica and allied genera. Japan F. P. Breeding, 19, 350-356. 